\title{
Movimientos sociales y reconfiguración de la democracia en el Siglo XXI ${ }^{1}$

\author{
Entrevista de \\ Daniela Cáceres a Geoffrey Pleyers ${ }^{2}$
}

A partir del 2010 se ha desarrollado un ciclo de movilizaciones sociales a escala global. Irrupciones como la primavera árabe, ocuppy’s, el 15-M en las plazas españolas, o sin ir más lejos la movilización estudiantil del 2011 en Chile, dan cuenta de que algo ocurre. Junto con ello los significantes: indignación, malestar, esperanza e imaginación se inscribieron en nuestro lenguaje público. Esta serie de manifestaciones se ha caracterizado por los cambios en las formas de organización, colaboración y participación, es que conjunto la sociedad ha cambiado, también los propios actores han revestido transformaciones respecto a los del siglo XX. Cambios que se expresan tanto en la creatividad de sus repertorios de acción y en las formas en las que hacen pública su crítica, como en las problemáticas que denuncian y las demandas que defienden. Además su capacidad les ha permitido articular escalas, pasando de lo local a lo global y viceversa. De esta manera, hoy la democracia es cuestionada y puesta en práctica a la vez, solo que no mediante las formas tradicionales a las cuales estamos habituados/as.

Por lo anterior, y en consideración a que la sociología de los movimientos sociales es uno de los ejes temáticos sobre los cuales

Los artículos de Geoffrey Pleyers están disponibles en: www.uclouvain. academia.edu/GeoffreyPleyers. Esta entrevista fue realizada por Daniela Cáceres, Académica e Investigadora de la Escuela de Sociología de la Universidad Católica Silva Henríquez. Contacto: dcaceresp@ucsh.cl.

Para los trabajos de edición y corrección de estilo al español se contó con la colaboración de Natalia Miranda, investigadora y estudiante de doctorado en CriDIS, Universidad Católica de Lovaina, Bélgica.

2 Entrevista realizada en el $9^{\circ}$ Congreso Chileno de Sociología, Universidad Católica del Maule, Chile. 
nuestra revista recibe contribuciones, no podíamos dejar pasar la oportunidad de entrevistar a uno de los más destacados exponentes en la actualidad. Geoffrey Pleyers, Doctor en sociología, académico de la Universidad Católica de Lovaina, Presidente del Comité de Investigación sobre movimientos sociales de la Asociación Internacional de Sociología (ISA) y miembro de nuestro comité internacional, fue invitado como uno de los conferencistas magistrales a la novena edición del Congreso Chileno de Sociología, realizado en el año 2016 en la ciudad de Talca.

Pleyers ha estudiado el movimiento altermundista, el zapatismo en México y las experiencias asociadas a la lógica del "buen vivir", proponiendo un nuevo paradigma para comprender los movimientos sociales. Su propuesta despliega la hipótesis de que existe una mutación en las formas de participación y en los actores sociales, fundamentalmente a raíz de la concepción que estos tienen del cambio social. De esta manera, los actores no necesariamente pretenden trasladar sus demandas a la escena política -institucional- a nivel nacional o internacional, sino que más bien, orientan su acción a un cambio social centrado en la sociedad, los activistas, los ciudadanos y las organizaciones locales.

Por ello, desde una reconfiguración de la democracia, no reducida a la política representativa e institucional, el cambio social se desarrolla en coherencia con las perspectivas que los actores tengan de este, en virtud de lo cual el autor propondrá, la "vía de la subjetividad" que privilegia la experiencia, entendiendo el cambio como "proceso", y, la "vía de la razón" que promueve la ciudadanía crítica e informada, así como también el rol que cumplen las y los intelectuales públicos -y/o la crítica científicafrente a las políticas dominantes, todo ello en pos de la construcción de un mundo más democrático.

Sobre esto y otros temas, entre conferencias, grupos de trabajo y sesiones de discusión, conversamos con Pleyers durante el Congreso realizado, como decíamos, en octubre de 2016 en Talca. 
Daniela Cáceres: Cuéntanos, ¿cuáles son los temas en que trabajas y aquellos que vienes analizando en profundidad?

Geoffrey Pleyers: Desde el año 2000 estoy trabajando en la línea de movimientos sociales de cara a la globalización. Primero con el movimiento alter-mundialista, que va desde los zapatistas -movimientos más comunitarios- a movimientos mucho más individualizados pero solidarios al mismo tiempo, como los jóvenes "alter-activistas". Desde el 2011 y de manera más reciente, me he enfocado en otros movimientos ciudadanos que apuntan a la democratización, entre ellos: España 15-M, Occupy Wall Street, Occupy Londres, las marchas en Brasil del 2013, y las protestas en Moscú.

Paralelamente, he estado investigando sobre las distintas formas que toma el movimiento ecologista en el Ecuador a partir del "buen vivir", hasta prácticas más individuales pero que también son colectivas, como el tema del consumo crítico, la manera de reducir el consumo, la disminución de su impacto ecológico desde prácticas cotidianas, así como la manera de trasladarse o de alimentarse. Finalmente, otra línea de trabajo corresponde a la epistemología de la investigación sobre los movimientos sociales, principalmente sobre los desafíos que enfrenta este campo en la actualidad.

DC: Sobre esto último, en cuanto a tus aspiraciones sobre la sociología de los movimientos sociales, ¿te interesa constituir una articulación teórica de diversas regiones? ¿Cuáles son tus proyecciones?

GP: La sociología de los movimientos sociales es un campo muy interesante, pues en ella se reflejan muchos de los grandes desafíos de la sociología contemporánea. Esto incluye la necesidad de no desatender las grandes preguntas teóricas y sociales, las cuales frecuentemente han sido abandonadas por los sociólogos, y al mismo tiempo, de no descuidar el trabajo de campo. Como sociólogos, no podemos concentrarnos únicamente en una escala micro-sociológica. También se debe vincular con una teoría de la sociedad. 
En ese sentido, una de las grandes preguntas de nuestro tiempo sería: ¿qué es la democracia en el siglo XXI? Sabemos que no basta tener elecciones para que la sociedad sea democrática, y que, a su vez, la globalización ha limitado la capacidad de decisión de los actores nacionales en varios asuntos. Al mismo tiempo, ciudadanos y actores de distintos movimientos sociales han establecido organizaciones democráticas tanto a nivel local como regional, en las cuales los activistas se juntan para analizar y difundir información por Internet, donde los movimientos no solo expresan sus deseos de un mundo más justo y democrático, sino que también experimentan elementos de ese otro mundo posible a partir de prácticas concretas. Así, observar y analizar la manera en que los actores de movimientos sociales, los ciudadanos y las comunidades cuestionan la monopolización de la democracia por parte de los partidos políticos y la política institucional, intentando a su vez implementar prácticas más democráticas y más horizontales, es una situación muy interesante, la cual contiene lecciones que van mucho más allá de estos grupos.

Por otra parte, también pienso que no debemos encerrarnos solamente en los estudios de casos. Debemos trabajar sobre ambas dimensiones -teórica y empírica-, , puesto que los actores se encuentran inventando un mundo nuevo hoy mismo, y repentinamente los sociólogos vamos quedando detrás de esa creatividad. De hecho, muchos de los grandes debates que han nutrido las ciencias sociales, han surgido hace algunas décadas dentro de los mismos movimientos sociales, como es el caso del feminismo, las luchas por el reconocimiento de derechos y los movimientos indígenas sobre asuntos ecológicos y en su cuestionamiento a la hegemonía de la cosmovisión moderna y occidental de la democracia y de lo que significa el "vivir bien". Todos estos debates surgieron en los movimientos sociales antes de difundirse en la filosofía política y las ciencias sociales. Por lo tanto, es importante considerar los actores de movimientos sociales como productores de prácticas, pero también como productores de conocimientos y visiones de mundo. 
Desde allí, la sociología y en particular la sociología de los movimientos sociales, aprende "de y con los actores", en un diálogo permanente con ellos. Sin embargo, no se trata de hacer una apología de cada movimiento y actor. No debemos olvidar que, ante todo, somos intelectuales críticos e investigadores con un rigor epistemológico y metodológico.

Por otro lado, la sociología global también me parece algo muy importante y un gran desafío hoy en día; más que un desafío, es una oportunidad extraordinaria.

\section{DC: ¿La sociología global entendida cómo...?}

GP: El primer elemento de una sociología global es ir más allá de lo que Ulrich Beck llamaba el "nacionalismo metodológico" pero sin caer en la trampa inversa, que denomino el "globalismo metodológico". El marco nacional no es siempre el enfoque que nos permite entender de mejor manera la realidad actual. A menudo, ver las cosas a partir de este marco nacional es precisamente lo que no nos permite entenderlas. Si bien los Estados siguen siendo actores importantes y la escala nacional se mantiene como relevante, no son centrales en todos los asuntos. A su vez, la investigación en ciencias sociales queda habitualmente establecida a esta escala, ya sea para recolectar los datos, entregar estadísticas y categorías de análisis, e incluso cuando se interpretan los datos. De esta manera, la globalización representa una gran oportunidad en términos de facilitar la movilidad de los investigadores, así como al acceso a los datos y resultados de investigaciones realizadas en otros países y continentes.

Una sociología más global nos puede ayudar a entender lo que está pasando en nuestro país o en nuestra región. Sin embargo, no hay que dejarse engañar por la idea inversa y considerar que todos los procesos sociales ocurren de la misma manera en todos los países, y que por lo tanto todo sería global de la misma manera desde lo que yo llamo "globalismo metodológico". Muchos intelectuales han publicado sobre la globalización de mane- 
ra muy general, leyendo los mismos periódicos globales y casi sin hacer trabajo de campo. A su vez, han masificado la idea de que el nivel local es solo el reflejo de dinámicas globales, lo que recuerda el título de un libro publicado hace 20 años: El fin de los territorios. Esto no puede estar más equivocado. Hoy somos testigos de lo erradas que estaban estas ideas. Con el agotamiento de los recursos naturales, las empresas extractivistas, los megaproyectos como Hidroaysén o las luchas por la soberanía alimentaria, vemos la pujante centralidad que tienen los territorios en lo que Martin Albrow ${ }^{3}$ llamó "Era Global".

Existen tres "antídotos" para "pensar global" sin caer en la trampa del globalismo metodológico.

El primero, como lo señalé, es combinar perspectivas más teóricas con trabajo de campo. Es indispensable tener una perspectiva global, de las evoluciones del "sistema-mundo" -como dirían algunos-, y de no limitar las ciencias sociales a las interacciones directas y al nivel micro. Pero también es indispensable anclar el trabajo y su análisis sobre los datos empíricos, es decir, sobre un sólido trabajo de campo. Hoy en día tenemos la posibilidad de hacer trabajos de campo en diferentes sitios ("multisituados") y a distintas escalas, lo que nos ayuda a entender los fenómenos y los actores globales.

El segundo antídoto corresponde a la apertura a un diálogo intercultural, a perspectivas distintas de los saberes hegemónicos que se han formulado en el Norte del planeta. Esto corresponde a lo que varios autores han llamado la "Epistemología del Sur": un cuestionamiento sobre nuestra manera de ver y entender el mundo, de nuestras categorías analíticas a partir de los actores sociales de otras regiones del mundo y de un diálogo intercultural, para retomar la formulación de Raúl Fornet-Betancourt. En mi caso, como hombre blanco occidental, observo el mundo con categorías occidentales. Por lo tanto, un diálogo con las Epis-

Albrow, M. (1996). The Global Age. Cambridge: Polity. 
temologías del Sur me ayuda no solo a entender la realidad de comunidades y ciudadanos del Sur del planeta, sino que también sobre lo que está pasando en el Norte del globo. Hoy en día, los investigadores del Sur tienen una contribución importante que hacer a la sociología internacional, no solo para explicar lo que está pasando en sus propios países, sino también para analizar y contribuir a un mejor entendimiento de la realidad social y de los desafíos en el Norte del planeta a partir de estos marcos. Por lo mismo, espero que más sociólogos latinoamericanos, asiáticos y africanos contribuyan a la sociología global a partir de sus investigaciones, ideas y perspectivas. Espero también no se limiten únicamente a analizar la realidad del Sur, sino que también a realizar investigaciones y análisis de lo que está pasando tanto en países europeos como norteamericanos.

El tercer antídoto es precisamente articular las escalas de acción y de análisis, de lo local a lo global. Volviendo a los movimientos sociales, vemos que, en un mundo profundamente globalizado, las principales propuestas alternativas han venido desde movimientos muy anclados en su territorio local pero que al mismo tiempo tienen alcances globales, conectándose con asuntos globales y presentando respuestas a algunos de los desafíos de la Era Global. Hoy en día, el movimiento más global y con mayor cantidad de miembros alrededor del planeta es la Vía Campesina, movimiento que alberga 200 millones de miembros de pequeños campesinos, el cual nos muestra cómo los campesinos tienen miles de soluciones frente al cambio climático, es decir, al desafío más global que estamos enfrentando actualmente. También están los movimientos indígenas, como los zapatistas en México o los Mapuches en Chile, quienes están proponiendo otras maneras de vivir y de organizarse democráticamente, así como de relacionarse con la comunidad y con la naturaleza, los cuales si bien están anclados en una comunidad local, tienen alcances globales.

Hasta ahora, la sociología de los movimientos sociales y la sociología en general siempre habían considerado los movimien- 
tos locales como poco importantes, resumiéndoles a acciones en defensa de intereses propios y egoístas de los habitantes de algún barrio (los movimientos "NIMBY", Not In My BackYard o "No en mi patio"). Estos eran considerados como actores que se quedaban en la escala local porque no tenían la fuerza suficiente para alcanzar el nivel nacional, siendo esta última la escala donde tanto politólogos como sociólogos consideraban que se jugaban las cosas importantes, es decir, donde los actores sociales buscaban influir sobre los gobiernos y los actores representativos. Los movimientos recientes no solo nos han enseñado la importancia del territorio y del espacio local en un mundo globalizado, sino también, que muchos actores no invierten tanta energía en una estructuración nacional, lo cual no ocurre por falta de fuerza $u$ organización, sino que porque consideran que volver a dar más importancia a la comunidad local y a los territorios puede contribuir a resolver problemas locales pero también globales, como es el caso del cambio climático, y al mismo tiempo, permitir a los ciudadanos recobrar la capacidad de decisión sobre su vida y su manera de organizarse. Aunque se mantengan en este nivel, estos movimientos locales tienen un alcance global, pues finalmente proponen alternativas al modelo de desarrollo y a la globalización neoliberal.

Personalmente, pienso que uno de los movimientos que va a ser muy importante en el mundo y en particular en América Latina es el movimiento del "buen vivir", el cual, a partir de vivencias, cuestiona la idea de lo que es una "vida buena", o en otras palabras, lo que es la felicidad. Lo que se nos vende es que para ser feliz "hay que tener dos automóviles, consumir mucho, tener una casa grande, irse lejos de vacaciones, y trabajar mucho". Eso se decía que era la felicidad. Y luego aparece un movimiento que dice “¡no!, esa no es la vida buena. El buen vivir o la vida buena es otra cosa, es una relación de no competición, pero sí de armonía con la naturaleza. Es una relación en la que mi entorno es importante, estar con la gente que quiero, con la familia, construir comunidad". Es otro concepto. Está cuestionando un ele- 
mento medular de nuestra sociedad: que nosotros en particular, como occidentales, vamos camino allí como si eso fuera lo lógico, como si esa fuera la única manera mediante la cual podemos ser felices, y esto no es algo obvio. Veo, por ejemplo, que muchos jóvenes ecologistas, así como otros que no se dicen ecologistas, están cuestionando esto mismo. Es más, he escuchado hablar del "buen vivir" desde otros caminos; por ejemplo, que es lo mismo realizar un trayecto en auto que con una "bici", que es importante consumir menos, que la felicidad está mucho más en las relaciones sociales que en tener más cosas y ser "siempre más rico". Existe un pensamiento muy importante en todo el mundo, un pensamiento cultural, que pasa por la subjetividad de cada uno; un modelo que no es únicamente de desarrollo, sino que se trata sobre la manera de "ser" en este mundo, que es el modelo de las subjetividades.

DC: En el fondo, tiene que ver con lo político y la política, o sea, cómo lo político está vivo en estas organizaciones comunitarias; por ejemplo, en estas organizaciones que son locales, pero que no tienen una interlocución directa como para disputar el poder en términos de la política tradicional.

GP: Se trata de cambiar la manera de interactuar con los demás. Los intelectuales y analistas plantean seguido que los movimientos de hoy "no tienen la fuerza para llegar al nivel político", o que "el movimiento estudiantil no fue suficientemente fuerte para transformarse en un partido político y cambiar la arena electoral". Por el contrario -y esto lo observé claramente en el movimiento "Noche en Pie" en París-, lo que es tan interesante en estos movimientos es precisamente que cuestionan la centralidad de la política institucional, que se oponen a la monopolización de la política por parte de políticos profesionales, y que afirman que la política es cosa de todos, lo cual también depende de los actos de cada uno de los ciudadanos, que el cambio lo hacemos nosotros y no los políticos, o mejor dicho, no solamente ellos. Pues lo que plantean es que mientras para algunos la democracia representativa no funciona, para otros esta no basta. 
Ahora bien, personalmente no niego el rol que juega la democracia representativa y la política institucional. Pero esta es solo una parte de la democracia, no su único escenario. En ese sentido, si algunos activistas de los movimientos de protesta recientes se pasan a la política institucional, no se trata de juzgar esto ni como un éxito ni como un fracaso. Probablemente puedan contribuir a un cambio progresista, o a reducir las desigualdades. Sin embargo, no debemos olvidar que la democracia representativa constituye solo una parte del escenario democrático, así como una de tantas fuerzas necesarias para promover y realizar transformaciones sociales.

\section{DC: Es solo una dimensión de la democracia...}

GP: Es una dimensión que se debe combinar con muchas otras. La democracia también se juega en nuestra vida cotidiana, en nuestra manera de vincularnos con los demás, en nuestras maneras de consumir. ¿Y es que acaso nuestra capacidad de acción y nuestra responsabilidad frente a problemas como el cambio climático o la pobreza se concentran únicamente en el voto? ¡Claro que no! También somos ciudadanos cuando consumimos, cuando nos trasladamos, o cuando elegimos cómo usar el dinero que ahorramos. Estos actos también tienen consecuencias en la comunidad, y por tanto, contribuyen a un tipo de sociedad. Así, en países como Francia o Chile donde existe un foco de atención alto sobre el gobierno nacional, las elecciones presidenciales y la política tradicional, me parece que los movimientos que cuestionan el monopolio de la democracia por parte de los actores de la política institucional son especialmente significativos.

La política no se puede resumir en un largo debate sobre quién va a ser la candidata o el candidato de tal coalición para luego tener una campaña electoral donde el marketing electoral y las frases asesinas predominen. ¿Sería esto la democracia? Pues no. Esta parte correspondería más a la sociedad del espectáculo de Guy Debord. Las campañas electorales son pensadas como un momento de politización. Pero tenemos que preguntarnos si no 
es al revés y si no son dominadas por procesos de despolitización. Primero, porque habitualmente se trata más de marketing y de impacto mediático que de debate con profundidad. Segundo, porque es un proceso donde se considera que lo político se va a delegar a una elite y a una esfera especializada dentro de la política institucional, cuando en realidad lo político está en todos los ámbitos de la vida. Por lo tanto, me parecen centrales aquellos movimientos que surgen planteando que lo clave quizás no es eso, sino que lo que se trata es de hacer cosas, que sea tanto la gente como los políticos quienes realizan los cambios.

\section{DC: Para construir un ejercicio democrático.}

GP: Hay que entender que estos ejercicios democráticos no se reducen a una manera de tomar decisiones colectivas. La democracia está también en la vida cotidiana. Muchos movimientos que mueven las sociedades de hoy no tratan tanto de cambiar la política, sino de cambiar la vida misma, como lo dijeron los zapatistas en México. La política es una parte de la vida. Hay que cuestionar la separación entre un "espacio público" que sería el lugar de lo político y una "vida privada" donde no se trata de política, como lo dijeron las feministas.

Por ejemplo, en la perspectiva que yo llamo "la vía de la subjetividad ${ }^{4 \prime}$, los activistas consideran que el cambio comienza por uno mismo. "Sé el cambio que quieres ver en el mundo" decía Gandhi. Frente al cambio climático, mucha gente comenzó a cuestionar y transformar su manera de consumir, de trasladarse, incluso lo que comen y el agua que gastan al bañarse. Son actos personales y muy locales, pero que también conectan a la persona con desafíos globales y transforman la subjetividad personal, y a partir de allí, la subjetividad colectiva.

4 Pleyers, G. (2010). Alter-Globalization. Becoming actor in the global age, Cambridge: Polity; Pleyers, G. (2015). Volverse actor: Dos vías para los movimientos sociales en el siglo XXI, Revista de Estudios Sociales, n54, p. 178-185 http:/ / res.uniandes.edu. co/view.php/1037/index.php?id=1037 
Ahora bien, no digo que estos desafíos globales se puedan resolver solo desde la vida cotidiana y las acciones locales. Nuevamente, la política no es algo que esté fuera de la vida o de lo cotidiano. Está inmersa en ella. El Estado, los actores de la política institucional también tienen un rol importante para limitar el cambio climático y la catástrofe ecológica, por ejemplo, regulando a los actores económicos. En consecuencia, tenemos que pensar estas distintas formas políticas como complementarias.

La batalla de la información es igualmente un frente muy importante en la defensa de la democracia hoy en día. Informarse es un compromiso de cada uno, pero también ver cómo difundir información verídica... Esta batalla se está dando desde varios frentes. Un primer frente es la crítica al poder de la información por parte de los grandes medios, en particular de los que pertenecen a los grandes empresarios. A pesar de la existencia de Internet, los grandes medios todavía tienen un poder increíble para dar forma a opiniones y subjetividades. Por lo tanto, es importante que existan redes ciudadanas que analicen la información oficial, la verifiquen y den a conocer perspectivas basadas sobre un análisis crítico.

DC: ¿Y qué término utilizarías para indicar ese momento/ espacio invisible desde el ojo público?

GP: Quizás política subterránea. O más bien, utilizaría sencillamente el término "política", porque para mí es parte de la política y de lo público.

\section{DC: ¿Por qué es público?}

GP: Porque se trata de la vida en común, y la vida en común no se juega solo en la televisión, los periódicos y los medios que a menudo pertenecen a las elites económicas. Se considera que es "político" solo si el tema llega y se debate en los grandes medios y por los actores de la política institucional. Esa idea responde a la lógica de una monopolización de la política por parte de una elite. Reducir la democracia y el espacio público a este aspecto es una trampa. 
Uno de los aspectos más interesantes de muchos movimientos actuales es que están implementando formas diferentes de democracia en todos los ámbitos de la vida. Lo que se está disputando a nuestros ojos, es una reconfiguración de la democracia para el siglo XXI.

Muchos activistas me dicen que están en contra de la democracia representativa, lo que se entiende muy bien, pero por mi parte pienso que la democracia representativa y las elecciones son elementos relevantes de la democracia en el siglo XXI, pero solo una parte.

DC: De hecho, muchas veces los activistas no utilizan el concepto de "democracia" y más bien se identifican y constituyen "formas alternativas a...".

GP: Pienso que una sociedad más democrática requiere la articulación de múltiples formas y prácticas de democracia. Veo en todos lados movimientos que no solo reivindican más democracia, sino que la implementan dentro de los espacios de los movimientos y en su vida personal misma.

De hecho, en todos lados se habla de una "crisis de la democracia". No estoy de acuerdo con este diagnóstico. Creo que lo que es visto como una crisis, y que a su vez corresponde a una insatisfacción profunda con la manera en que está organizada la democracia hoy en día, es un elemento permanente en ella. Y el hecho de que tantos jóvenes reivindiquen e implementen más democracia me parece muy positivo, y en parte un éxito para ella.

Por otro lado, tampoco estoy de acuerdo con el discurso de la "post-democracia", como lo plantea Colin Crouch. El politólogo inglés explica que la democracia está declinando desde su auge en los años '70, cuando entonces el sistema político estaba muy integrado a nivel nacional, mientras que ahora, la democracia formal se ha extendido, pero ha perdido mucho de su sustancia ya que los gobiernos electos han perdido mucha capacidad de acción y de decisión. 
Primero, creo que no hay que caer en una idealización del mundo de los años '70. Esto parece obvio en Chile. Una buena parte de los europeos vivían también en dictaduras, sea bajo el régimen soviético o en regímenes fascistas como en España, Portugal o Grecia. Asimismo, la democracia norteamericana llevó la guerra a Vietnam y apoyó golpes de Estado en América Latina.

Segundo, hay que reconocer que si bien ha habido retrocesos de la democracia en Europa, como el poder de los sindicatos, también hemos avanzado en otras cosas; por ejemplo, en muchos países de Europa se impusieron límites mucho más fuertes al financiamiento de los partidos políticos por parte de las empresas.

Y tercero, muchos actores se encuentran movilizados con el objetivo de extender las prácticas y exigencias de la democracia en todos los ámbitos de la vida. Existen una multitud de grupos, redes y movimientos que promueven una participación ciudadana, un decrecimiento del consumo, la igualdad de género...

Sin embargo, también es cierto que a pesar de la extensión de la democracia formal y de prácticas democráticas en varios ámbitos de la vida, se ampliaron las desigualdades y surgieron otros obstáculos en el camino hacia sociedades más democráticas.

DC: Desde un punto de vista metodológico: ¿cómo podría articularse una propuesta de investigación que nos permita observar los movimientos sociales en la sociedad actual?

GP: Necesitamos nuevas herramientas analíticas, heurísticas, y reinventar nuestras maneras de ver los actores sociales. Creo que conceptos como experiencia vivida, compromiso personal o subjetivación son centrales para entender los movimientos de hoy. Muchos de los actores que se levantaron desde el 2011 lo hicieron a partir de un compromiso muy personal y ético, desde su vida, valores y subjetividad, con la voluntad de relacionarse con los demás de una manera no utilitarista, desarrollando relaciones interpersonales sin jerarquías ni estatus. 
Bajo esta perspectiva y sin caer en un "globalismo metodológico" que sería mantener la idea de que los movimientos "post2011" fueron idénticos en todos los países, es interesante apuntar a una cultura política compartida por una parte de los jóvenes movilizados en muchos países, desde el movimiento estudiantil en Chile hasta "Noche en Pie" en Francia, pasando por Occupy en Nueva York, el movimiento de Gezi en Turquía, y el de los paraguas en Hong Kong. Adoptan un activismo muy individualizado y personal pero a la vez solidario. Todos denunciaron la colusión de los grandes medios con el poder político y económico, e implementaron formas más participativas de democracia dentro de sus organizaciones y en las plazas ocupadas.

DC: $Y$ en este análisis, ¿tiene algo más que agregar respecto de Chile?

GP: Quizás lo que a mí más me impactó durante esta segunda estancia en Chile - la primera fue hace dos años- es la capacidad del sistema para mantenerse y reproducirse a pesar de ser deslegitimado por un movimiento tan fuerte e interesante como fue el movimiento estudiantil del 2011.

Durante los primeros años que siguieron al movimiento de 2011, se publicaron artículos y libros que sostenían que el "modelo chileno" estaba roto, que el movimiento estudiantil le había quitado toda su legitimidad. Pero pese a la fuerza y a la amplitud del movimiento, este modelo sigue siendo muy poderoso todavía. Muchos hemos subestimado la capacidad del sistema de mantenerse y de proponer soluciones que permiten mantener la matriz socio-política vigente. El sistema de la política institucional aspiró una parte de las energías contestatarias, integrando unos líderes estudiantiles y alterando las demandas del movimiento para que se pueda responder con una ley que no cambia esencialmente el sistema de educación superior.

Sin embargo, como dije antes, sería un error resumir el alcance del movimiento a su impacto en la política institucional. El movimiento de 2011 también transformó a las personas que 
participaron en él y abrió nuevos espacios, probablemente un nuevo ciclo de protesta desde la deslegitimación al modelo neoliberal en Chile. La meta de los movimientos sociales es romper el sentido común, cuestionar las orientaciones dominantes de la sociedad. Así fue el caso de este movimiento, que cuestionó el "sentido común" de que la sociedad neoliberal es justa y que se basa en la meritocracia, cuando esto en realidad no es cierto. Si bien su impacto en la educación superior fue menor de lo que se esperaba, es a partir de las marchas masivas donde comienza la deslegitimación del modelo, desarrollando prácticas alternativas que se enraizaron en canales menos visibles, en las redes sociales, y en la manera de organizarse en la universidad. El cuestionamiento y la crítica al modelo neoliberal no se quedó en el marco de la educación superior, sino que en muchos asuntos de la vida de los chilenos. Y de ahí surge el movimiento "No más AFP", que lo podríamos visualizar como una continuación de la crítica profunda al modelo iniciada el 2011. 